\title{
Persistence and compliance with osteoporosis therapies among postmenopausal women in the UK Clinical Practice Research Datalink
}

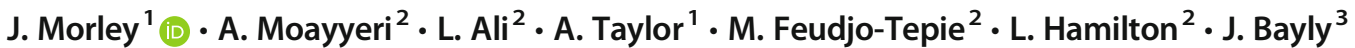

Received: 5 February 2019 / Accepted: 6 November 2019 / Published online: 22 November 2019

(C) The Author(s) 2019

\begin{abstract}
Summary Gaining full benefits from osteoporosis medications requires long-term treatment. Investigating the real-world persistence of women receiving osteoporosis medications in the UK, we found that most patients stop treatment within a year. To prevent osteoporotic fragility fractures, long-term treatment persistence must be improved.

Introduction Persistence with osteoporosis therapies has historically been poor. To treat this chronic and progressive disease, it is essential that patients receive the full benefit of these medications. We estimated persistence and compliance with osteoporosis therapies in a large sample of postmenopausal women in the UK.

Methods Data were obtained from the Clinical Practice Research Datalink for all women aged 50 years and over or women with early menopause, who received at least one prescription in primary care for any licensed osteoporosis therapy between January 1, 2010 and December 31,2015. Persistence and compliance at 24 months (primary objective) and at 5 years (exploratory objective) were estimated in three patient cohorts: "All Patients," "Naïve Patients," and "Drug-Specific."

Results The All Patients cohort included 72,256 women. Persistence with any therapy was 56.1\%, 43.6\%, 36.4\%, and $31.0 \%$ at $6,12,18$, and 24 months, respectively, and $23.2 \%$ and $13.1 \%$ at 3 years and 5 years, respectively. Patients were generally more persistent and compliant if evaluated from their first exposure to osteoporosis therapy (Naïve Patients cohort). In the drug-specific analysis, 64\% of patients receiving denosumab (administered subcutaneously every 6 months) were persistent at 24 months compared with $28 \%$ and $23 \%$ of those taking oral bisphosphonates and intravenous bisphosphonates, respectively.

Conclusions Only about one in three patients who received osteoporosis therapy continued to be on treatment after 2 years. There is a need to improve persistence with osteoporosis therapy, especially for high-risk patients
\end{abstract}

Keywords Clinical Practice Research Datalink · Compliance $\cdot$ CPRD $\cdot$ Osteoporosis $\cdot$ Persistence $\cdot$ Postmenopausal

\section{Introduction}

Osteoporotic fragility fractures are one of the most disabling consequences of aging in women, being associated with significant morbidity and mortality and a reduction in quality of

Electronic supplementary material The online version of this article (https://doi.org/10.1007/s00198-019-05228-8) contains supplementary material, which is available to authorized users.

\section{J. Morley}

jmorley@amgen.com

1 Amgen Ltd., Cambridge, UK

2 Amgen Ltd., Uxbridge, UK

3 University of Derby, Derby, UK life [1]. In the UK, there are approximately 3.21 million people aged 50 years and over with osteoporosis [2]. There are approximately 536,000 new fragility fractures each year [2], and the lifetime risk of any fracture is estimated to be $53 \%$ at age 50 years among women [3]. The UK economic burden of new and prior fractures is estimated at $£ 4,397$ million each year, which is predicted to increase by $24 \%$ to $£ 5,466$ million by 2025 [2]. The impact of osteoporotic fractures is therefore far reaching, not only for individuals but also for the health service and economy.

A variety of effective pharmacological interventions are approved for patients with osteoporosis, including bisphosphonates, selective estrogen receptor modulators (SERMs), parathyroid hormone (PTH) peptides, and a receptor activator of nuclear factor kappa B ligand (RANKL) inhibitor (denosumab) [4]. For these medications to deliver 
the full potential benefit seen in clinical trials in a real-world setting, sufficient levels of both persistence and compliance with the approved regimen are necessary [5]. Persistence can be defined as the duration of time from initiation to discontinuation of therapy, while compliance can be defined as the extent to which a patient acts in accordance with the prescribed interval and dosing of a regimen [6]. Oral bisphosphonates, the most widely used treatment for osteoporosis, are associated with poor persistence and compliance. Studies have shown that up to $50 \%$ of patients discontinue oral bisphosphonates during the first year of treatment [7], and approximately $30-50 \%$ of patients do not take their medication as directed [8]. Low persistence and compliance are associated with a greater risk of osteoporotic fracture, which may increase morbidity and mortality $[5,9,10]$. Furthermore, poor persistence is associated with increased healthcare resource utilization $[9,11]$.

In the UK, published studies of persistence with osteoporosis therapy have extended only as far as 2008 , and have been limited largely to oral bisphosphonates, specifically alendronate and risedronate [12-15]. Given the approval of new medications in recent years, including denosumab and the parenteral bisphosphonates zoledronate and ibandronate (for which the dose frequency and mode of administration vary from the traditional bisphosphonate therapy), an up-todate UK analysis would be of benefit to the healthcare system to determine the characteristics of patients who receive osteoporosis therapy in primary care and to identify ways in which persistence could be improved.

The objective of this study was to estimate persistence and compliance with both oral and parenteral osteoporosis therapies in a large sample of postmenopausal women in the UK, who were treated in the primary care setting between 2010 and 2015. A secondary objective was to estimate the same parameters in subpopulations of these women who were treatmentnaïve or previously treated (defined as the non-naïve treatment group).

\section{Methods}

\section{Data resource}

This study was a retrospective analysis using the Clinical Practice Research Datalink (CPRD), a longitudinal database of anonymized medical records from UK primary care. The CPRD contains data on diagnoses, prescriptions, laboratory investigations, clinical outcomes, and hospital referrals for approximately 11.3 million patients from more than 670 representative primary care practices throughout the UK (as of January 2014) [16]. Further information on the CPRD can be found on the associated website [17]. The database is representative of the age, sex, and geographic distribution of the
UK population $[16,18]$. CPRD patients' data are linked to mortality records at the Office of National Statistics. Thus, the UK CPRD provides a valuable resource for evaluating persistence and associated patient characteristics and treatment patterns over time.

\section{Study population}

All women in the CPRD who received at least one prescription for a licensed osteoporosis therapy between January 1, 2010 and December 31, 2015 inclusive and were aged 50 years and over, or aged under 50 years experiencing a premature (including surgery-induced) menopause, at receipt of the initial prescription (i.e., the index date) were potentially eligible for inclusion in the study. Participants were required to have at least one full year of data in their record before the first receipt of osteoporosis therapy (i.e., the initial prescription) and to have at least 6 months of recorded data after the initial prescription. These requirements ensured that there were sufficient patient-level data to capture information on comedications and comorbidities and to estimate persistence for at least 6 months. Exclusion factors included a history of cancer (except non-melanoma skin cancer) or metabolic bone disease (including rickets or osteomalacia, hyperparathyroidism, and Paget disease of bone) before or on the day of the initial prescription.

The monitored therapies were classified into six categories: oral bisphosphonates (e.g., risedronate, alendronate, ibandronate, etidronate), parenteral bisphosphonates (e.g., zoledronate [formulations licensed for treatment of osteoporosis], ibandronate), SERMs (i.e., raloxifene), PTH (e.g., teriparatide, 1-84 PTH), strontium ranelate (which was approved during the study period, but is now no longer available in the UK [19]), and denosumab. Calcium, vitamin D, hormone replacement therapy, or related molecules (such as tibolone or sex steroid derivatives) as a sole treatment were not considered osteoporosis therapies (unless combined with recognized therapies).

Patients were evaluated from the date of the initial prescription recorded in the CPRD for 24 months or until discontinuation of osteoporosis therapy, within the observation period January 1, 2010 to December 31, 2015. Records for osteoporosis therapies during 2008 and 2009 were considered to identify previous exposure and windows of treatment for these drugs.

\section{Study cohorts}

Data from three patient cohorts were analyzed: "All Patients," "Naïve Patients," and "Drug-Specific" (Supplementary Figure 1). 


\section{All Patients cohort}

This cohort consisted of all patients initiating an osteoporosis therapy during the observation period. Each patient had one prescription window, from the index date of the first osteoporosis therapy to the date of discontinuation of any osteoporosis therapy, which could include more than one therapy. Discontinuation/end of therapy was defined depending on the time interval between the adjacent prescriptions. If the gap between the expected end date covered by the previous prescription and the next prescription date was greater than 30 days, the patient was assumed to have discontinued therapy and their end of therapy was the end date of the previous prescription. If the patient was exposed to any osteoporosis therapies before the start of the observation period (i.e., January 1, 2010), the start of the first prescription window after January 1, 2010 was used as her index date (i.e., there had to be a gap of at least 30 days in her treatment during the observation period to be eligible for inclusion in the cohort).

\section{Naïve Patients cohort}

This cohort was a subgroup of the All Patients cohort and consisted of those patients initiating osteoporosis therapy during the observation period that had no previous osteoporosis therapy recorded. Those who had received any osteoporosis therapy in the 12 months before 2010 were excluded from this cohort. Each patient's prescription window was defined as for women of the All Patients cohort.

In the All Patients and Naïve Patients cohorts, data were analyzed in a patient-centric manner, in which persistence with osteoporosis medication was measured irrespective of changes in drug class.

\section{Drug-Specific cohort}

In this cohort, data were analyzed in a drug class-specific manner to assess the importance of drug class on persistence, for either starting or subsequent treatment. For identifying prescription windows, changes of drug within a class (e.g., oral bisphosphonates) were included in the same window. If a patient changed drug class (e.g., from oral to parenteral bisphosphonates), even without a 30-day gap, her first window would be closed and a new window for the second drug class would be started. A gap of more than 30 days was required between the old and new therapy from the same drug class for a new window to be started.

Naïve treatment and non-naïve treatment windows were assessed in the Drug-Specific cohort. Naïve treatment windows were those in which patients initiated an osteoporosis therapy during the observation period who had no exposure to any osteoporosis treatments within 12 months of the start of the observation period. The treatment window was from the index date of the first osteoporosis therapy to the end of the same osteoporosis therapy, and the window could only include one type of medication. Non-naive treatment windows were those in which patients initiated an osteoporosis therapy during the observation period after having received a previous osteoporosis therapy. The window was from the date of the new osteoporosis therapy to the end of osteoporosis therapy with the same drug class. As a patient could receive multiple drug classes during the observation period, she could have several non-naïve treatment windows (but only one naïve treatment window).

\section{Persistence and compliance}

Persistence was quantified using the estimated level of persistence with therapy (ELPT) method, which determines the percentage of individuals remaining on therapy (i.e., persistent) at a given time [20]. It was calculated as the number of days from initiating osteoporosis therapy (i.e., index date) to end of therapy and was estimated as the proportion of patients refilling each subsequent prescription within the 30-day permissible gap. Patients not refilling within the permissible gap were considered nonpersistent. Calculations of persistence incorporated a "storage up" algorithm, which took into account the start date and durations of each prescription so that if a patient had overlapping prescriptions, she would be considered persistent until the time that all existing prescriptions failed to cover the gap period (i.e., if a new prescription was issued before the end date of the previous prescription, the start date of the new prescription was shifted to the end of the previous prescription). The percentage of patients persisting at $6,12,18$, and 24 months was estimated as the primary study objective, with the percentage at 3 and 5 years estimated as an exploratory objective.

Compliance was quantified using the medication possession ratio (MPR) for oral therapies and medication coverage ratio (MCR) for parenteral therapies, as previously described [21]. The MPR was calculated as the sum of the days' supply of medication divided by the number of days between the first prescription and the exhaustion of the last prescription during the predetermined time windows. Women with only one prescription during the predetermined time windows were therefore excluded from this analysis. The MCR measured the percentage of days that the patient was covered by a long-acting agent over a given time interval after receiving the injection. Compliance (MPR or MCR) was calculated for the subset of patients who remained persistent at $6,12,18$, and 24 months (primary objective) and 3 and 5 years (exploratory objective); at each time point, patients who were not persistent were excluded from the compliance analysis. 
A sensitivity analysis of the persistence and compliance measures was performed using alternative permissible gaps of 60,90 , and 120 days.

\section{Baseline characteristics}

Demographic and clinical characteristics of all included patients were collected from their medical records from the 12month period before the index date. History of prior fracture at baseline was defined as any recorded fracture (except fractures of fingers, toes, face, or skull) in the 5-year period before the index date. Estimates of 10-year risk of major osteoporotic fracture (MOF) and hip fracture were calculated using the Fracture Risk Assessment Tool (FRAX 4.0, FRAX, University of Sheffield, Sheffield, UK).

\section{Statistical analysis}

All data summaries were descriptive in nature. For categorical variables, the frequency and percentage were given. Mean and standard deviation measures were reported for continuous variables. Kaplan-Meier curves were used to visualize persistence probabilities. Treatment discontinuation was treated as the failure event; data were censored at patient death or date of last follow-up. Statistical analyses were performed using SAS version 9.4 (SAS Institute, Cary, NC, USA).

\section{Results}

\section{Study population}

The total number of potentially eligible patients for the All Patients and Naïve Patients cohorts was 102,486 (Fig. 1a). After exclusion of patients with a history of cancer, metabolic bone disease, or fewer than 6 months of follow-up, the final study populations of the All Patients and Naïve Patients cohorts were 72,256 and 46,924 patients, respectively. A slightly higher number of patients, 103,436, were eligible for the Drug-Specific cohort (because this cohort included patients who entered the observation window on treatment and switched drug within a 30-day gap period); after exclusions, this cohort consisted of 72,857 patients (Fig. 1b). Within this cohort, 46,390 patients were observed in naïve treatment windows and 44,039 patients were observed in non-naïve treatment windows.

The baseline characteristics of the patients in the three cohorts are summarized in Table 1 and Supplementary Tables S1, S2, and S3. Oral bisphosphonates were by far the most common starting treatment (All Patients cohort 68,400/

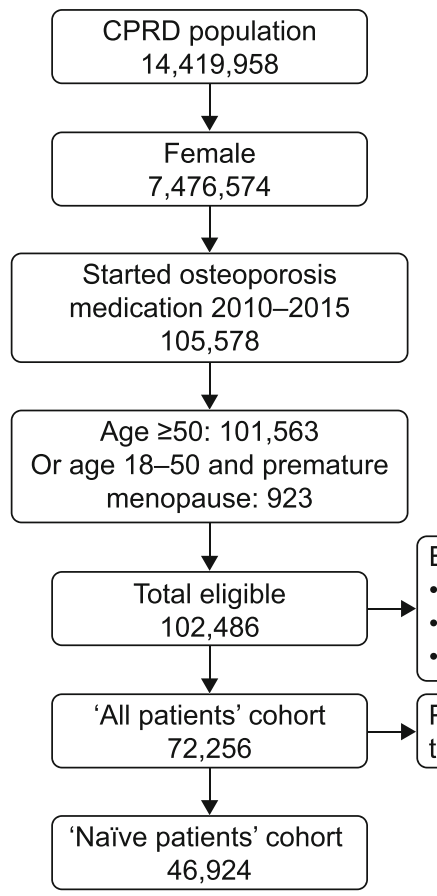

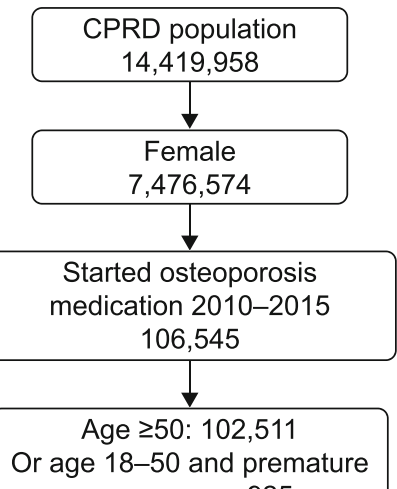

menopause: 925

Excluded due to:

- history of cancer: 22,021

metabolic bone disease: 1694

- <6 months of follow-up: 9712

Patients with previous OP

treatment excluded

'Drug-specific' cohort 72,857
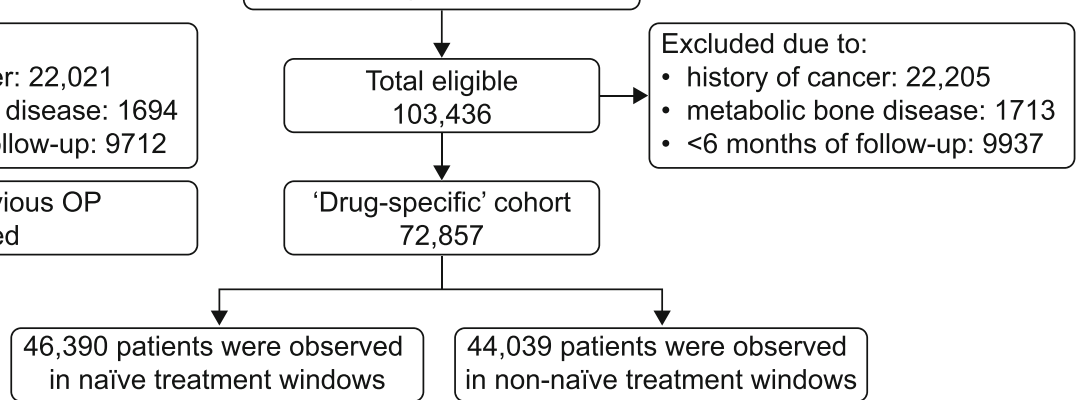

multiple drug classes during the observation period. Each patient could therefore have several non-treatment-naïve windows but only one treatment-naïve windowTables and figures 
$72,256[94.7 \%]$ ) reflecting therapeutic guidelines [4]. By contrast, very few patients were prescribed parenteral bisphosphonates $(38 / 72,256[0.05 \%])$ or PTH $(35 / 72,256$ $[0.05 \%])$. In the All Patients cohort, the mean age of women who received strontium (77.0 years), denosumab (76.9 years), or PTH (75.6 years) was higher than those receiving bisphosphonates (oral, 74.0 years; parenteral, 73.4 years) or SERMs (68.8 years) (Table 1).

The majority of patients did not have a diagnosis of osteoporosis recorded. Denosumab and PTH users were more likely to have a diagnosis $(39.7 \%$ and $42.8 \%$, respectively, in the All Patients cohort) compared with the overall study population (29.4\% of the total All Patients cohort). Furthermore, most patients had no history of fracture; only $35.1 \%$ of women in the All Patients cohort had a history of fracture. The proportion of women with a history of fracture was higher in patients receiving PTH (51.4\%) or denosumab (49.9\%) than in those receiving other therapies, particularly SERMs (16.1\%). Almost no patients had a Read code indicating a familial history of hip fracture. The FRAX-calculated 10-year risk of major osteoporotic fracture was higher in patients receiving denosumab (All Patients cohort 22.45\%), strontium $(21.87 \%)$, or PTH $(21.24 \%)$ than in those receiving other treatments (13.81-19.48\%).

Baseline characteristics were generally similar between patients of the Drug-Specific cohort observed in naïve and nonnaïve treatment windows (Supplementary Tables S2 and S3). Patients generally had a slightly higher risk of fracture at the time of their initial (naïve) treatment versus their subsequent (non-naive) treatment. The proportions of patients who died during the study period while receiving each medication were as follows: oral bisphosphonates, $5.5 \%(3836 / 69,413)$; strontium, 4.4\% (209/4712); denosumab, 4.4\% (50/1141); parenteral bisphosphonates, $4.1 \%$ (3/73); SERM, 1.9\% (13/671); and PTH, $1.8 \%(1 / 56)$.

\section{Persistence and compliance: all osteoporosis medications}

Kaplan-Meier analysis showed that persistence with osteoporosis medication (any class) among women of the All Patients cohort decreased over time; persistence was $56.1 \%$ at 6-month follow-up and decreased to $31.0 \%$ at 24 months (Fig. 2). Only $13.1 \%$ of evaluable women in the All Patients cohort were still persistent at 5 years. Persistence was higher among women of the Naïve Patients cohort than the All Patients cohort at all time points (Naïve patients $62.1 \%$ at 6 months, $37.3 \%$ at 24 months; Fig. 2). Median duration of persistence was 8.5 months in the All Patients cohort and 12.4 months in the Naïve Patients cohort.

Compliance measures were generated for patients who remained persistent at the beginning of each 6-month time interval within the observation period. Compliance with 
Fig. 2 Kaplan-Meier analysis of discontinuation with any class of osteoporosis medication in the "All Patients" and "Naïve Patients" cohorts using a 30-day permissible gap

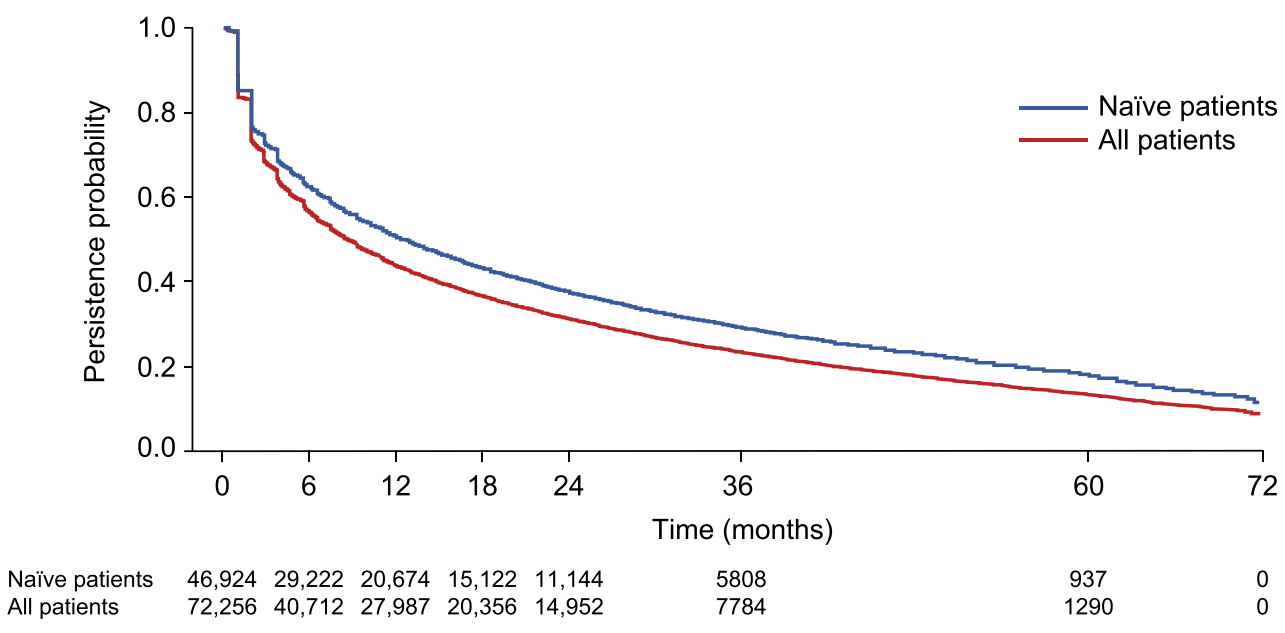

osteoporosis medication (any class) in the All Patients and Naïve Patients cohorts was lowest during 0 to $<6$ months and increased during each time interval to its highest rate at 18 to $<24$ months (Supplementary Table S4). At each time interval, compliance was slightly higher in the Naïve Patients cohort than in the All Patients cohort.

\section{Persistence and compliance: drug class-specific}

In the Drug-Specific cohort, Kaplan-Meier analysis showed that persistence at 24 months was higher with denosumab than with any other therapy; persistence rates were $50.0 \%$ for denosumab compared with $19.0 \%$ for oral bisphosphonates, $15.2 \%$ for parenteral bisphosphonates, $12.9 \%$ for SERMs, $6.9 \%$ for strontium, and $0.0 \%$ for PTH (Table 2, Fig. 3). Parenteral bisphosphonate persistence was high at 6 months, at $100 \%$, but decreased to $22.7 \%$ at 18 months and $2.7 \%$ at 5 years. In the same period, $70.1 \%$ of patients on denosumab were still persistent at 6 months, which decreased to $57.0 \%$ at 18 months and to $16.1 \%$ at 5 years. Persistence with oral bisphosphonates decreased from $39.7 \%$ at 6 months to $22.8 \%$ at 18 months and to $7.8 \%$ at 5 years.

Median duration of persistence was higher with parenteral therapies than with oral therapies; median persistence was 24.0 months for denosumab and 12.0 months for parenteral bisphosphonates compared with 3.7 months for oral bisphosphonates, 2.5 months for SERMs, 1.9 months for strontium, and 0.9 months for PTH (Fig. 3).

Compliance with parenteral therapies was higher than with oral therapies (Supplementary Table S4).

\section{Naïve vs non-naïve persistence and compliance}

Persistence with oral medications at 6, 12, 18, and 24 months was higher in naïve treatment windows than non-naïve treatment windows (Table 2, Fig. 4), which was particularly apparent for oral bisphosphonates $(37.7 \%$ persistence at 24 months for naïve treatments vs $11.3 \%$ for non-naïve treatments). Median persistence duration with oral bisphosphonates was 12.9 months in naïve treatment windows and 2.6 months in non-naïve treatment windows (Fig. 4). By contrast, persistence with parenteral bisphosphonates was higher in non-naïve treatment windows than in naive treatment windows at all time points. Although, there was no difference in median duration of persistence between naïve and non-naïve treatment windows for patients receiving parenteral bisphosphonates (12.0 vs 12.0 months). Patients receiving denosumab were more likely to remain persistent if prescribed this therapy in nonnaïve treatment windows than in naïve treatment windows (median persistence duration in months 25.4 vs 12.6).

Compliance with oral medications was generally higher as naïve treatments than non-naïve treatments. Conversely, compliance with parenteral medications was generally higher as non-naïve treatments than as naïve treatments (Supplementary Table S4).

\section{Sensitivity analysis}

When re-estimating persistence using longer permissible medication refill gaps of 60,90 , and 120 days (vs the original 30 days), persistence increased with the length of gap, as expected (Supplementary Table S5). Increasing the length of gap appeared to have a greater effect on increasing persistence with oral drugs (e.g., oral bisphosphonates and strontium) compared with injectable drugs (parenteral bisphosphonates and denosumab) over the 24 months of follow-up. A similar effect was observed for compliance, but the effect was reduced after 12 months of follow-up (Supplementary Table S4).

\section{Discussion}

In this first real-world UK study examining persistence and compliance with osteoporosis therapies in postmenopausal 


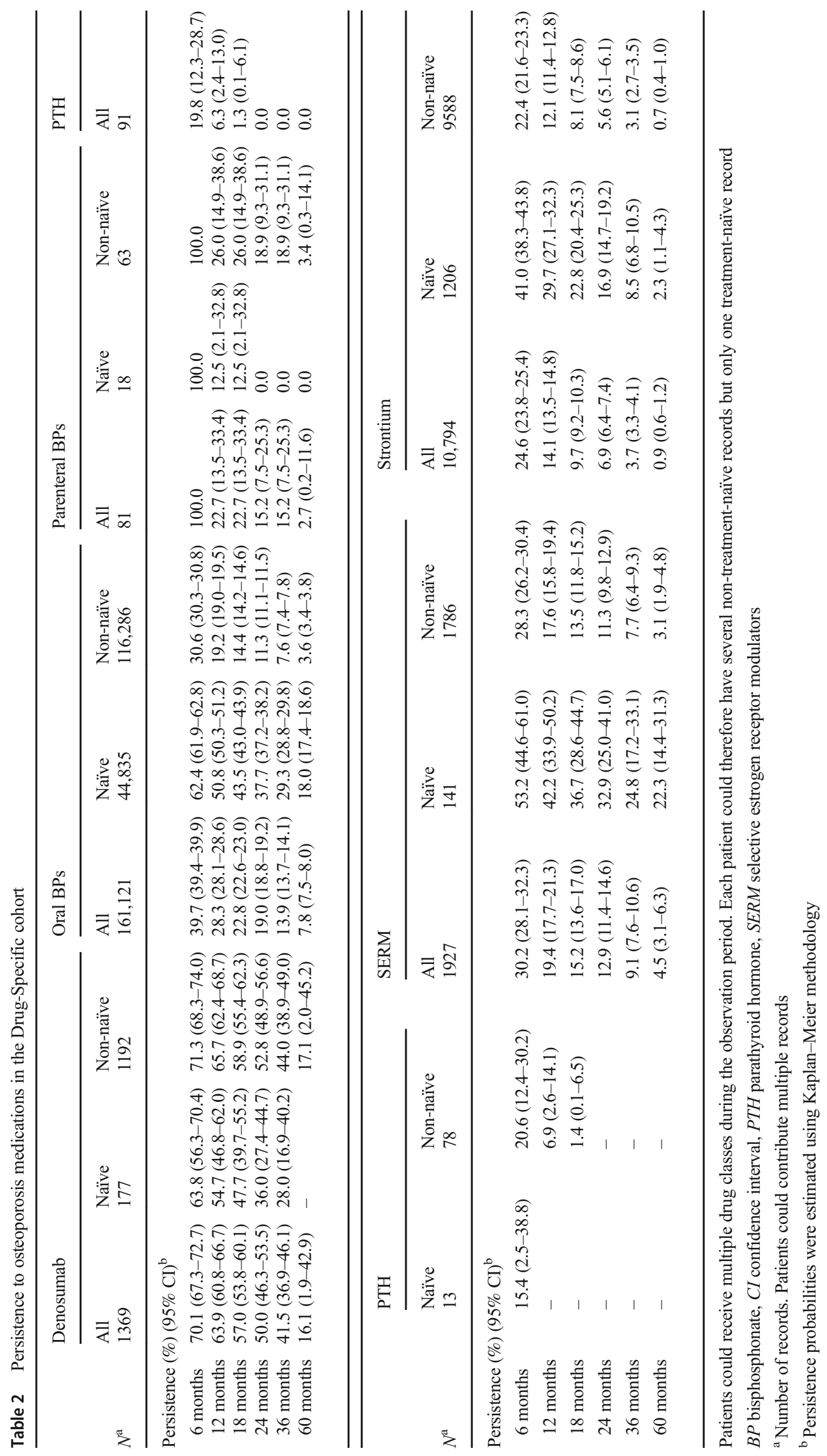




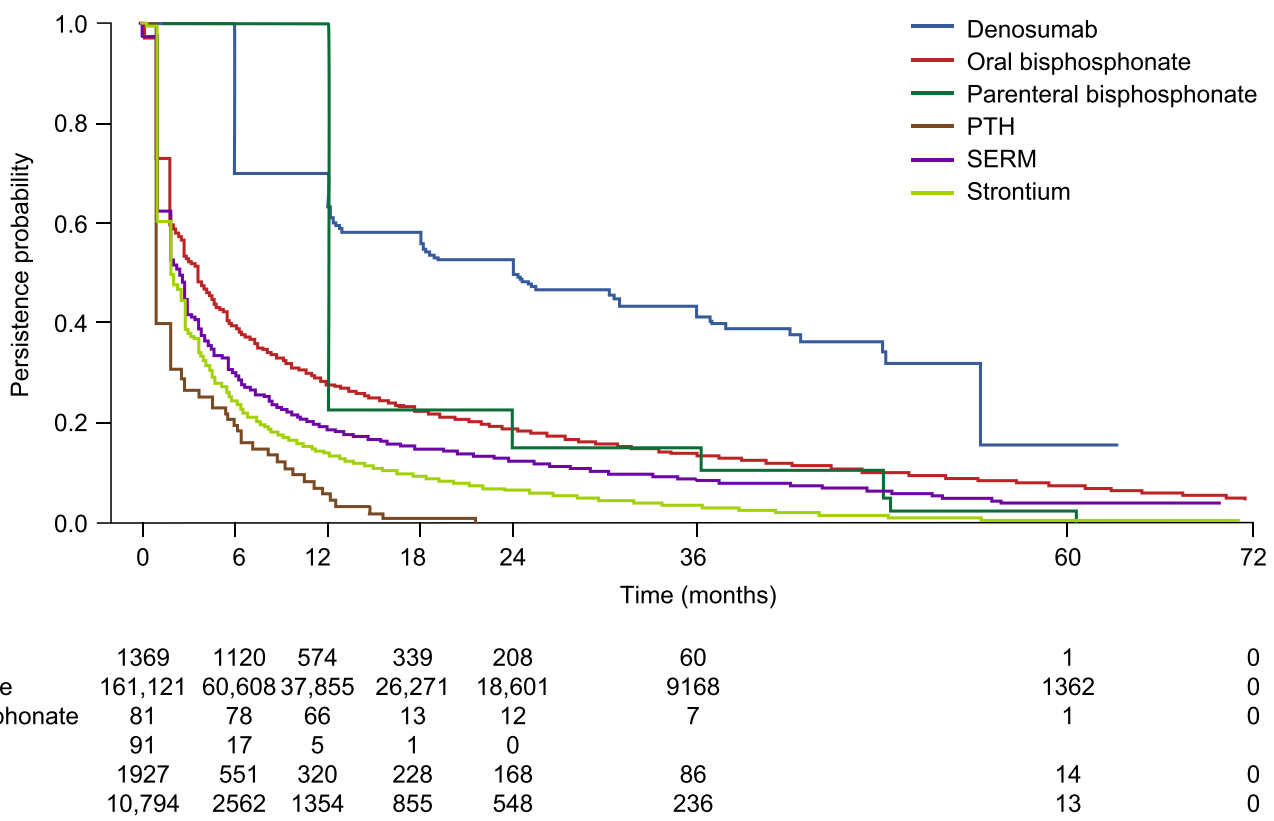

Fig. 3 Kaplan-Meier analysis of discontinuation with each medication class over a 5-year period in the "'Drug-specific' Specific" cohort, using a 30-day permissible gap. Patients could contribute multiple records. The numbers at risk for each medication represent the number of records not the number of patients. PTH parathyroid hormone, SERM selective estrogen receptor modulators women since 2008, we found that persistence with all osteoporosis medications remains poor, with only about one in three patients who started treatment remaining persistent at 24 months. In the longer term, our study identified that just over $10 \%$ of UK patients remain persistent after 5 years of treatment. Patients were generally more persistent if evaluated from their first exposure to osteoporosis therapies. Drugspecific analysis showed that the median duration of persistence was higher with parenteral therapies than with oral therapies. Furthermore, persistence at 24 months was higher with denosumab than with any other therapy. These results should be placed in the context of guidance from the National Osteoporosis Guideline Group (NOGG) for recommended durations of therapy for osteoporosis medications, which would have been in place for part of this study [4], and the fact that the CPRD captures treatment recorded in the primary care setting only should be noted.

Low persistence with prescribed medication regimens is regarded as a major problem in the treatment of osteoporosis, with consequences of increased fracture risk $[5,10]$. Indeed, a previous UK database analysis comparing fracture risk in patients with current versus past use of oral bisphosphonates found that at least 6 months of active treatment was required to reduce the risks of hip/femur fracture and osteoporotic fracture. For hip fracture, the greatest risk reduction for patients with a current versus those with a past use of bisphosphonates occurred after 2 years of treatment, and the risks of fracture were increased by low compliance [13]. Our results highlight the finding that a substantial proportion of patients do not persist with treatment for long enough to gain its expected benefit and that few persist for long enough for the NOGG guideline on medication review to be relevant [4]. It should be noted, however, that NOGG guidance (revised in 2013) states that patients should have their osteoporosis treatment reviewed every 3 or 5 years (intravenous bisphosphonates and oral bisphosphonates, respectively) and that a treatment holiday should be considered at this time [22]; persistence data for these agents collected after 2013 could have been influenced by physicians discontinuing medications in response to the guidelines.

At the time of the previous CPRD persistence study (19952008), denosumab (administered subcutaneously) had not yet been approved in the European Union, and zoledronate (administered intravenously) had only recently been approved and so would not have had adequate data collected during this observation window [12-15]. Parenteral osteoporosis therapies have the potential to improve persistence and compliance compared with oral bisphosphonates because they have a longer duration of action, eliminating the requirement to take a tablet daily or weekly [21]. In this study, we found that the median duration of persistence was higher among users of parenteral treatments than oral treatments. The very low persistence $(0 \%)$ at 24 months reported here for parenterally administered PTH is expected, given that its label requirements stipulate a maximum total duration of treatment of 24 months [23] and that it is frequently administered in a specialist or secondary care setting. At almost every time point, persistence was higher with denosumab than with any other therapy when analyzed using a permissible gap of 30 days. Although longer permissible gap periods can be used for the analysis of persistence for parenteral 
therapies (e.g., denosumab or zoledronate) because the frequency of prescription for these drugs is typically lower and patients may not receive them as regularly as oral drugs, sensitivity analysis using permissible gaps of 60,90 , and 120 days confirmed the results of the 30-day analysis.

Previous persistence studies of denosumab covering the USA, Canada and several European countries excluding the UK have reported persistence at 12 months of 55.9-99.1\% and at 24 months of 34.8-99.5\% [24-35]. In contrast, for other parenteral therapies, the previous studies reported persistence at 12 months of $33.8-74.5 \%$, and for oral therapies reported 10-78\% [21, 28]. At 24 months, reported persistence was $20.9-35.8 \%$ for parenteral therapies and $15.9-63.5 \%$ for oral therapies [24, 26-28]. Our data show similarly high persistence with denosumab at 12 and 24 months $(63.9 \%$ and $50.0 \%$, respectively). Surprisingly, in this study, persistence with oral bisphosphonates was slightly higher than that with parenteral bisphosphonates at both $12(28.3 \%$ vs $22.7 \%)$ and 24 months (19.0\% vs $15.2 \%)$.

Patients in this study were generally more persistent to oral treatments if they had no exposure to osteoporosis therapies in the previous 12 months (i.e., were naïve treated). Initial exposure to a medication class resulted in a generally longer duration of treatment compared with second or later windows of treatment (i.e., non-naïve treatments). This indicates that patients tend to discontinue treatment if they have previously dropped out of treatment. National Institute for Health and Care Excellence (NICE) accredited guidelines recommend the use of oral bisphosphonates for first-line prevention in the majority of patients [4]. If a patient switches to a different therapy, this may be due to gastrointestinal adverse effects, physician recommendation, and lack of adherence owing to frequent dosing $[9,14]$. For parenteral therapies, however, there was a greater likelihood of persistence in non-naïve treatment windows than in naïve treatment windows. This is in line with results from a previous database study, which showed that persistence remained suboptimal following switching to a second therapy and further declined after a switch to a third or further treatment; however, switching from an initial osteoporosis therapy to another, preferably with a lower frequency of administration, may increase persistence [15]. These data may also suggest that a patient's perceived disease severity influences their persistence [36]; patients prescribed denosumab were more likely to have had a diagnosis of osteoporosis, a previous hip fracture, and a higher 10-year probability of fracture than those prescribed other therapies and may, therefore, be more motivated to remain persistent than patients with less severe disease. Patient preference for parenteral therapies may also influence persistence; data from a randomized-controlled crossover trial showed that persistence and compliance increased when patients switched from alendronate to denosumab, but decreased when patients switched from denosumab to alendronate [24].
Our study provides an up-to-date analysis (for the period 2010-2015) of persistence and compliance with osteoporosis treatment in UK primary care, building on previous studies using the CPRD database (formerly General Practice Research Database [GPRD]) [12-15]. Brankin et al. evaluated bisphosphonate therapies including alendronate and risedronate from 2001 to 2005 assessing the difference in compliance and persistence between weekly and daily regimens across three different UK data sets [12]. Results from the GPRD data set showed that those on weekly regimens had higher compliance (MPR) than those on daily regimens, and they persisted longer with treatment. A second study using the GPRD, by Gallagher et al., evaluated the persistence of bisphosphonate therapy including alendronate and risedronate from 1987 to 2006 [13]. Results showed that $58.3 \%$ of the patients continued bisphosphonate treatment for $>1$ year and $23.6 \%$ for $>5$ years. Finally, Li et al. examined GPRD data from the period 1995-2008, using similar methods to our study (including a 30-day permissible medication refill gap) [14]. Approximately $18 \%$ of daily alendronate users were persistent at 1 year, with a similarly poor rate of around $41 \%$ of weekly alendronate users being persistent after this time. By 3 and 5 years of follow-up, non-persistence rates were over $90 \%$ for most osteoporosis therapies studied. In the present study, both short- and long-term persistence with any osteoporosis medication was higher compared with the rates observed by Li et al. (All Patients cohort vs Li et al. "All Women" cohort 6 months, $56.1 \%$ vs $44.2 \%$; 12 months, $43.6 \%$ vs $32.2 \%$; 3 years, $23.2 \%$ vs $16.0 \%$; 5 years, $13.1 \%$ vs $9.3 \%$ ). Higher persistence in the first year of treatment observed in the present study may be due to closer monitoring of patients earlier in their treatment, as part of improved management of osteoporosis in accordance with recent NICE and NOGG treatment guidelines $[4,37]$.

Various interventions have been trialed to help patients adhere to osteoporosis therapy, such as patient education, patient monitoring, and simplification of dosing regimens, although most have had limited success [38]. The introduction in the late 1990s of Fracture Liaison Services (FLSs) in the UK might be expected to have influenced persistence and compliance with therapy as has been demonstrated with FLSs in other countries; however, data on long-term follow-up are limited [39]. Moreover, the introduction in April 2012 of a Quality and Outcomes Framework (QOF) domain for secondary fracture prevention within primary care might also be expected to have improved persistence [40]. Despite these developments, we found that overall persistence with osteoporosis medication in 2010-2015 has only increased marginally since the Li et al. study of 1995-2008 [14]. The penetration of FLS at a patient level is acknowledged to be patchy and variable [41], and furthermore, the success of the QOF osteoporosis domain has been limited in its first 2 years, with as few as one in five of the expected number of eligible patients with a fragility fracture 


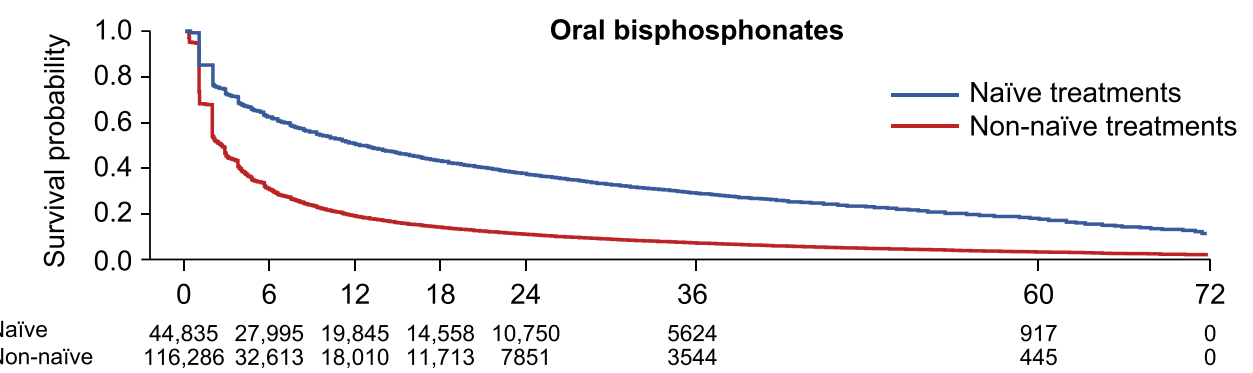

$\begin{array}{lllllll}\text { Non-naïve } & 116,286 & 32,613 & 18,010 & 11,713 & 7851 & 3544\end{array}$
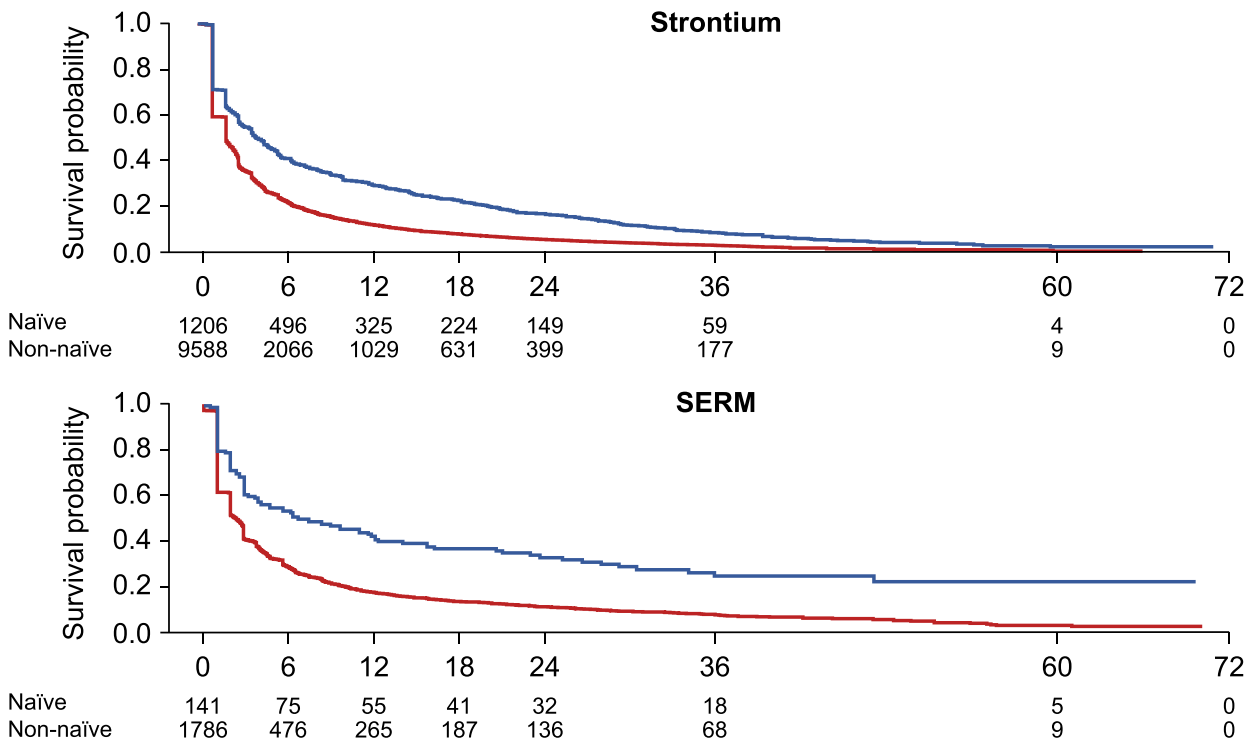

$1.0, \quad$ Parenteral bisphosphonate

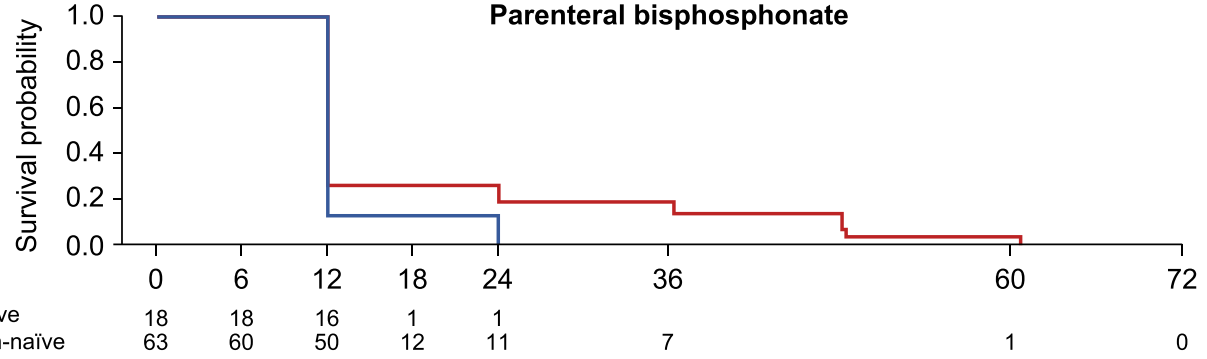

$\begin{array}{lllllllll}\text { Non-naïve } & 63 & 60 & 50 & 12 & 11 & 7 & 1 & 0\end{array}$

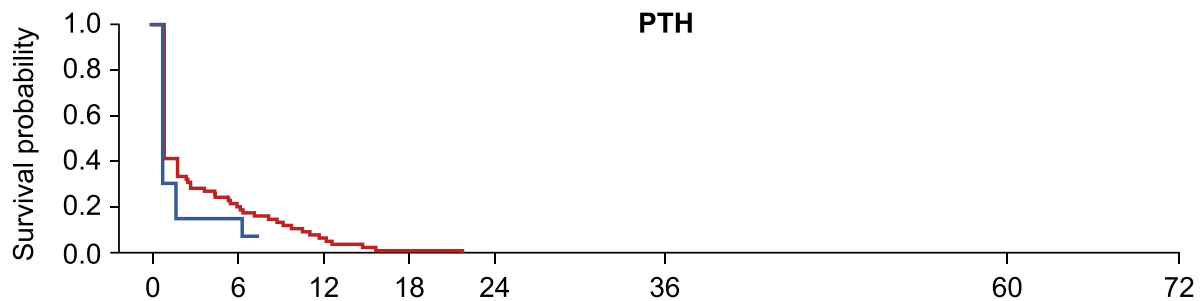

$\begin{array}{llllll}\text { Naïve } & 13 & 2 & 0 & \\ \text { Non-naïve } & 78 & 15 & 5 & 1\end{array}$

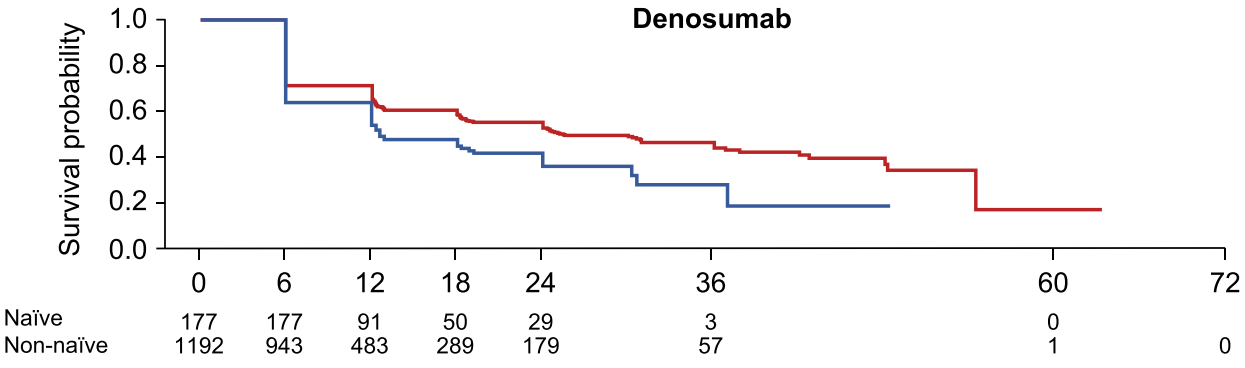

Time (months) 
Fig. 4 Kaplan-Meier analysis of discontinuation with each medication class over a 5-year period in the "'Drug-specific"' cohort (naïve vs nonnaïve treatments), using a 30-day permissible gap. PTH parathyroid hormone, SERM selective estrogen receptor modulators

receiving appropriate care [42]. The introduction of FLSs may have had some benefit in improving persistence, but this is limited beyond 12 months of treatment [43].

Although all patients in this study were receiving osteoporosis medication, less than one third had a recorded osteoporosis diagnosis. This indicates either that healthcare providers are prescribing preventive medication before osteoporosis is diagnosed or are failing to add the diagnosis to electronic health records. We also observed a low history of fracture prevalence in the previous 5 years, suggesting that most osteoporosis treatments are being directed at primary prevention. A recorded diagnosis of osteoporosis and history of prior fracture appeared to be more common among patients receiving denosumab than in those receiving other medications. This may be due to patients being prescribed denosumab after previously receiving oral therapies. NICE guidelines prioritize the use of denosumab for secondary prevention, and potential reasons for switching to denosumab could include a high risk of fracture, inability to comply with dosing instructions, intolerance, or contraindications to treatments used in primary prevention [44].

A Canadian cohort study found that osteoporosis treatment (bisphosphonates, raloxifene, calcitonin, systemic estrogen replacement therapy) only reduced the actual fracture rate below that predicted by FRAX among patients in the highest tertile of risk who were persistent for 5 years with high adherence [45]. A US claims database study showed fracture rates in the overall population were reduced following treatment with bisphosphonates, denosumab, and teriparatide, but rates in patients with previous exposure to osteoporosis medications were reduced only in denosumab and teriparatide-treated patients. Additionally, the magnitude of reduction was greater in patients with a prior fracture than those without [46]. These data indicate that patients should be appropriately targeted for treatment, with consideration given to baseline fracture risk and previous treatment history and with emphasis on ensuring optimal treatment and persistence in patients with a high-risk of fracture.

The main limitation of this study is that only treatments recorded in primary care are captured in the CPRD. Compared with other therapies, PTH and parenteral bisphosphonates are more likely to be administered in secondary care and hence may not have been recorded adequately in the CPRD. Denosumab is prescribed in primary care, but its initiation in primary or secondary care varies by region. Therefore, the duration of persistence measured in this study will be underestimated for medications initially prescribed in secondary care. Conversely, it is possible that patients transferred from secondary to primary care were selected for based on an increased likelihood they would remain persistent.
Finally, given that only treatments recorded in primary care were captured in the CPRD, this may have led to limited numbers of patients in some drug classes (e.g., PTH, parenteral bisphosphonates, and denosumab), although this may be reflective of clinical practice. Additionally, we only collected data on patients who stopped therapy, were lost to follow up or died while on therapy, and did not record reasons for treatment discontinuation; some patients may have discontinued therapy in line with clinical guidance such as that from the NOGG. Another limitation is that although all postmenopausal women receiving anti-osteoporotic therapy during the study period were identified, some younger women with surgical or chemotherapy-induced menopause may have been missed if their treatment or procedures occurred before their registration date in the CPRD. Limited information on parental fracture history is recorded in the CPRD, so this factor would have been set to zero in the FRAX tool, possibly leading to underestimation of 10-year fracture probability. Finally, we analyzed data according to treatment status (i.e., naïve and nonnaïve treated), but only 12 months of medical history were required before entry into the study. There could, therefore, have been instances in which a patient completed a course of osteoporosis therapy before this 12-month period yet still met the criteria for being treatment-naïve. Despite these limitations, the CPRD provides a good representation of the general UK population of postmenopausal women, recording extensive prescription data, and has the benefit of providing longterm follow-up information, although it should be noted that we could follow only a limited number of patients for the full 5 -year exploratory objective as the observation period was 6 years in length (January 1, 2010 to December 31, 2015).

In conclusion, persistence with osteoporosis therapy remains poor in UK primary care. Owing to the chronic and progressive nature of osteoporosis, patients need to persist with therapy to gain the clinical benefit from their medication. Current guidelines suggest treatment for 3-5 years with fracture risk reassessed after this time [4]; however, it is apparent from the current analysis that many patients discontinue their treatment after 12-24 months. There is a major need to improve persistence and compliance with osteoporosis medication.

Acknowledgments Medical writing support was provided by Richard Claes, PhD, of Oxford PharmaGenesis, Oxford, UK.

Funding information This study was funded by Amgen Ltd.

\section{Compliance with ethical standards}

Conflicts of interest JM, AT, and MF-T are employees of Amgen and hold stock in Amgen. LA is an independent consultant contracted by Amgen to undertake this work. AM and LH were employees of Amgen during the design, analysis, and initial drafting of the manuscript. JB received consultancy fees from Amgen for protocol development and data analysis. 
Open Access This article is distributed under the terms of the Creative Commons Attribution-NonCommercial 4.0 International License (http:// creativecommons.org/licenses/by-nc/4.0/), which permits any noncommercial use, distribution, and reproduction in any medium, provided you give appropriate credit to the original author(s) and the source, provide a link to the Creative Commons license, and indicate if changes were made.

\section{References}

1. Hernlund E, Svedbom A, Ivergard M, Compston J, Cooper C, Stenmark J, McCloskey EV, Jonsson B, Kanis JA (2013) Osteoporosis in the European Union: medical management, epidemiology and economic burden. A report prepared in collaboration with the International Osteoporosis Foundation (IOF) and the European Federation of Pharmaceutical Industry Associations (EFPIA). Arch Osteoporos 8:136

2. Svedbom A, Hernlund E, Ivergard M, Compston J, Cooper C, Stenmark J, McCloskey EV, Jonsson B, Kanis JA, EURPo IOF (2013) Osteoporosis in the European Union: a compendium of country-specific reports. Arch Osteoporos 8:137

3. van Staa TP, Dennison EM, Leufkens HG, Cooper C (2001) Epidemiology of fractures in England and Wales. Bone 29:517522

4. National Osteoporosis Guideline Group (NOGG). Clinical guideline for the prevention and treatment of osteoporosis. 2017. https:// www.sheffield.ac.uk/NOGG/NOGG\%20Guideline\%202017.pdf. Accessed 09 May 2019

5. Siris ES, Selby PL, Saag KG, Borgstrom F, Herings RM, Silverman SL (2009) Impact of osteoporosis treatment adherence on fracture rates in North America and Europe. Am J Med 122:S3-S13

6. Cramer JA, Roy A, Burrell A, Fairchild CJ, Fuldeore MJ, Ollendorf DA, Wong PK (2008) Medication compliance and persistence: terminology and definitions. Value Health 11:44-47

7. Cramer JA, Gold DT, Silverman SL, Lewiecki EM (2007) A systematic review of persistence and compliance with bisphosphonates for osteoporosis. Osteoporos Int 18:1023-1031

8. Cramer JA, Amonkar MM, Hebborn A, Altman R (2005) Compliance and persistence with bisphosphonate dosing regimens among women with postmenopausal osteoporosis. Curr Med Res Opin 21:1453-1460

9. Chodick G, Moser SS, Goldshtein I (2016) Non-adherence with bisphosphonates among patients with osteoporosis: impact on fracture risk and healthcare cost. Expert Rev Pharmacoecon Outcomes Res 16:359-370

10. Keshishian A, Boytsov N, Burge R, Krohn K, Lombard L, Zhang X, Xie L, Baser O (2017) Examining the effect of medication adherence on risk of subsequent fracture among women with a fragility fracture in the U.S. medicare population. J Manag Care Spec Pharm 23:1178-1190

11. Ferguson S, Feudjo Tepie M, Taylor A, Roddam A, Critchlow C, Iqbal M, Spangler L, Bayly J (2016) The impact of persistence with bisphosphonates on health resource utilization and fracture risk in the UK: a study of patient records from the UK Clinical Practice Research Datalink. J Eval Clin Pract 22:31-39

12. Brankin E, Walker M, Lynch N, Aspray T, Lis Y, Cowell W (2006) The impact of dosing frequency on compliance and persistence with bisphosphonates among postmenopausal women in the UK: evidence from three databases. Curr Med Res Opin 22:1249-1256

13. Gallagher AM, Rietbrock S, Olson M, van Staa TP (2008) Fracture outcomes related to persistence and compliance with oral bisphosphonates. J Bone Miner Res 23:1569-1575
14. Li L, Roddam A, Gitlin M, Taylor A, Shepherd S, Shearer A, Jick S (2012) Persistence with osteoporosis medications among postmenopausal women in the UK General Practice Research Database. Menopause 19:33-40

15. Li L, Roddam A, Ferguson S, Feudjo-Tepie M, Taylor A, Jick S (2014) Switch patterns of osteoporosis medication and its impact on persistence among postmenopausal women in the U.K. General Practice Research Database. Menopause 21:1106-1113

16. Herrett E, Gallagher AM, Bhaskaran K, Forbes H, Mathur R, van Staa T, Smeeth L (2015) Data resource profile: Clinical Practice Research Datalink (CPRD). Int J Epidemiol 44:827-836

17. Medicines and Healthcare products Regulatory Agency (2018) Welcome to Clinical Practice Research Datalink. https://www. cprd.com/home/ Accessed 15 March 2018

18. Jick SS, Kaye JA, Vasilakis-Scaramozza C, Garcia Rodriguez LA, Ruigomez A, Meier CR, Schlienger RG, Black C, Jick H (2003) Validity of the general practice research database. Pharmacotherapy 23:686-689

19. National Osteoporosis Society (2017) Osteoporosis drug strontium ranelate (Protelos) no longer available after August. https://nos.org. uk/news/2017/may/31/osteoporosis-drug-strontium-ranelateprotelos-no-longer-available-after-august/ Accessed 10 September 2018

20. Dezii CM (2001) Persistence with drug therapy: a practical approach using administrative claims data. Manag Care 10:42-45

21. Hadji P, Papaioannou N, Gielen E et al (2015) Persistence, adherence, and medication-taking behavior in women with postmenopausal osteoporosis receiving denosumab in routine practice in Germany, Austria, Greece, and Belgium: 12-month results from a European non-interventional study. Osteoporos Int 26:2479-2489

22. Compston J, Bowring C, Cooper A et al (2013) Diagnosis and management of osteoporosis in postmenopausal women and older men in the UK: National Osteoporosis Guideline Group (NOGG) update 2013. Maturitas 75:392-396

23. Gedeon Richter (2018) Teriparatide: summary of product characteristics https://www.ema.europa.eu/documents/productinformation/terrosa-epar-product-information_en.pdf Accessed 6 April 2018

24. Freemantle N, Satram-Hoang S, Tang ET, Kaur P, Macarios D, Siddhanti S, Borenstein J, Kendler DL (2012) Final results of the DAPS (Denosumab Adherence Preference Satisfaction) study: a 24-month, randomized, crossover comparison with alendronate in postmenopausal women. Osteoporos Int 23:317-326

25. Fuksa L, Vytrisalova M (2015) Adherence to denosumab in the treatment of osteoporosis and its utilization in the Czech Republic. Curr Med Res Opin 31:1645-1653

26. Hadji P, Kyvernitakis I, Kann PH et al (2016) GRAND-4: the German retrospective analysis of long-term persistence in women with osteoporosis treated with bisphosphonates or denosumab. Osteoporos Int 27:2967-2978

27. Karlsson L, Lundkvist J, Psachoulia E, Intorcia M, Strom O (2015) Persistence with denosumab and persistence with oral bisphosphonates for the treatment of postmenopausal osteoporosis: a retrospective, observational study, and a meta-analysis. Osteoporos Int 26:2401-2411

28. Lakatos P, Takacs I, Marton I, Toth E, Zoltan C, Lang Z, Psachoulia E, Intorcia M (2016) A retrospective longitudinal database study of persistence and compliance with treatment of osteoporosis in Hungary. Calcif Tissue Int 98:215-225

29. Papaioannou A, Khan A, Belanger A et al (2015) Persistence with denosumab therapy among osteoporotic women in the Canadian patient-support program. Curr Med Res Opin 31:1391-1401

30. Silverman SL, Siris E, Kendler DL et al (2015) Persistence at 12 months with denosumab in postmenopausal women with osteoporosis: interim results from a prospective observational study. Osteoporos Int 26:361-372 
31. Fahrleitner-Pammer A, Papaioannou N, Gielen E et al (2017) Factors associated with high 24-month persistence with denosumab: results of a real-world, non-interventional study of women with postmenopausal osteoporosis in Germany, Austria, Greece, and Belgium. Arch Osteoporos 12:58

32. Reyes C, Tebe C, Martinez-Laguna D, Ali MS, Soria-Castro A, Carbonell C, Prieto-Alhambra D (2017) One and two-year persistence with different anti-osteoporosis medications: a retrospective cohort study. Osteoporos Int 28:2997-3004

33. Migliaccio S, Francomano D, Romagnoli E et al (2017) Persistence with denosumab therapy in women affected by osteoporosis with fragility fractures: a multicenter observational real practice study in Italy. J Endocrinol Investig 40:1321-1326

34. Tremblay E, Perreault S, Dorais M (2016) Persistence with denosumab and zoledronic acid among older women: a population-based cohort study. Arch Osteoporos 11:30

35. Durden E, Pinto L, Lopez-Gonzalez L, Juneau P, Barron R (2017) Two-year persistence and compliance with osteoporosis therapies among postmenopausal women in a commercially insured population in the United States. Arch Osteoporos 12:22

36. McHorney CA, Gadkari AS (2010) Individual patients hold different beliefs to prescription medications to which they persist vs nonpersist and persist vs nonfulfill. Patient preference and adherence 4:187-195

37. National Institute for Health and Care Excellence (NICE). Osteoporosis: assessing the risk of fragility fracture. Clinical guideline [CG146]. Published August 2012. https://www.nice.org.uk/ guidance/cg146. Accessed 19 December 2017

38. Hiligsmann M, Salas M, Hughes DA, Manias E, Gwadry-Sridhar FH, Linck P, Cowell W (2013) Interventions to improve osteoporosis medication adherence and persistence: a systematic review and literature appraisal by the ISPOR Medication Adherence \& Persistence Special Interest Group. Osteoporos Int 24:2907-2918

39. Walters S, Khan T, Ong T, Sahota O (2017) Fracture liaison services: improving outcomes for patients with osteoporosis. Clin Interv Aging 12:117-127
40. National Health Service / British Medical Association. Quality and Outcomes Framework guidance for GMS contract 2013/14. March 2013. https://www.bma.org.uk/-/media/files/pdfs/ practical $\% 20$ advice $\% 20$ at $\% 20$ work/contracts/ gpqofguidance20132014.pdf Accessed 15 September 2017

41. Royal College of Physicians. Fracture Liaison Service Database (FLS-DB) clinical audit. FLS forward: identifying high-quality care in the NHS for secondary fracture prevention. April 2017. https:// www.rcplondon.ac.uk/projects/outputs/fls-db-clinical-auditidentifying-high-quality-care-nhs-secondary-fracture. Accessed 29 June 2018

42. Health and Social Care Information Centre. Quality and outcomes framework-prevalence, achievements and exceptions report: England, 2014-15. Published 29 October 2015. http://digital.nhs. uk/catalogue/PUB18887. Accessed 19 December 2017

43. Royal College of Physicians. Falls and Fragility Fracture Audit Programme (FFFAP). Fracture liaison service Database (FLS-DB) facility audit. May 2016. https://www.rcplondon.ac.uk/projects/ outputs/fls-db-facilities-audit-fls-breakpoint-opportunitiesimproving-patient-care Accessed July 2016

44. National Institute for Health and Care Excellence (2010) Denosumab for the prevention of osteoporotic fractures in postmenopausal women. https://www.nice.org.uk/guidance/ta204/ chapter/1-Guidance Accessed 6 April 2018

45. Leslie WD, Lix LM, Johansson H, Oden A, McCloskey E, Kanis JA (2012) Does osteoporosis therapy invalidate FRAX for fracture prediction? J Bone Miner Res 27:1243-1251

46. Yusuf AA, Cummings SR, Watts NB, Feudjo MT, Sprafka JM, Zhou J, Guo H, Balasubramanian A, Cooper C (2018) Real-world effectiveness of osteoporosis therapies for fracture reduction in post-menopausal women. Arch Osteoporos 13:33

Publisher's note Springer Nature remains neutral with regard to jurisdictional claims in published maps and institutional affiliations. 